\title{
El concepto de poder en las relaciones internacionales y la necesidad de incorporar nuevos enfoques
}

\author{
The concept of power in international relations and \\ the need of incorporating new approaches
}

Nicolás Creus*

\section{Resumen}

El propósito de este artículo es analizar las diferentes concepciones del poder presentes en la disciplina de las Relaciones Internacionales, poniendo de manifiesto la insuficiencia de los abordajes tradicionales y la necesidad de ampliar la perspectiva teórica. De este modo, se espera avanzar hacia una comprensión más profunda y acabada del concepto. Tal como lo advierten muchos autores, es menester romper con la tentación simplificadora de definir el poder exclusivamente en términos de la posesión de recursos. Un avance fundamental radica en reconocer su carácter relacional y explorar las diferentes dimensiones en que opera. La ampliación del horizonte teórico resulta tan necesaria como inevitable.

Palabras Clave: Poder - Teoría de las Relaciones Internacionales - poder como recurso - poder como relación - dimensiones del poder

Docente adscripto de «Política Internacional Argentina», Universidad Nacional de Rosario (UNR-Argentina).nicolascreus@gmail.com

Recibido el 7 de mayo de 2013; aceptado el 14 de junio de 2013 


\section{Abstract}

The purpose of this article is to analyse the different conceptis of power present in the discipline of International Relations, revealing the deficiencies of traditional approaches and the need for broadening the theoretical perspective. The paper thus tries to provide the tools to reach a thorough comprehension of the concept of power. As many authors remark, it is necessary to break with the simplifying temptation of defining power exclusively in terms of resources. An important step is to recognize its relational character and explore the different dimensions in which it operates. Broaden the theoretical horizons is as necessary as unavoidable.

KEYwORDs: Power - International Relations Theory - power defined as resources - power defined as relation - dimensions of power 


\section{INTRODUCCIÓN}

El poder constituye un concepto sumamente complejo, pero al mismo tiempo fundamental para explicar y comprender la dinámica de las relaciones internacionales. Tradicionalmente su estudio ha estado muy vinculado a los análisis de la escuela realista, en tanto que desde esta la política internacional se entiende fundamentalmente como una lucha constante por el poder. No obstante, en los últimos años se han suscitado numerosos debates y discusiones al respecto, que exigen ampliar el horizonte teórico e incorporar nuevos enfoques. Por esta razón, resulta conveniente analizar el concepto a la luz de las diferentes perspectivas teóricas presentes en la disciplina. Cada una de estas destaca y enfatiza diferentes aspectos y dimensiones del poder, arribando de tal forma a conclusiones igualmente diferentes sobre su naturaleza y sus usos.

Según Berenskoetter (2007:1), las formas de entender el poder «determinan cuáles actores y relaciones consideramos relevantes y dónde localizamos espacios políticos -en breve, cómo conceptualizamos la política mundial-, es pertinente por tanto estar atentos a las diferentes maneras en las cuales el poder puede ser definido». En tal sentido, el autor considera necesario «pensar sobre el poder».

En consonancia con estas apreciaciones, el presente trabajo se propone analizar las diferentes concepciones del poder presentes en la disciplina de las relaciones internacionales, poniendo de manifiesto la insuficiencia de los abordajes tradicionales y la necesidad de ampliar el horizonte teórico, a fin de poder avanzar hacia una comprensión más profunda y acabada del concepto. Además, la reflexión sobre el poder es fundamental no solo para desentrañar la complejidad de la política internacional sino también para el análisis y la formulación de la política exterior de un Estado, en tanto que permite evaluar desde una óptica más sofisticada los recursos disponibles y las posibilidades y formas de utilizarlos (Creus, 2011).

En función de las inquietudes referidas y la importancia del concepto en cuestión, en la primera parte del trabajo examinan las diferentes concepciones del poder en la perspectiva realista, entendiéndolas como punto de partida necesario para discutir sobre el poder en las relaciones internacionales. El segundo apartado se concentra en el debate entre la visión del poder entendido como sinónimo de la posesión de recursos y aquella que lo entiende como un concepto relacional. Luego, como consecuencia inmediata del citado debate, en la tercera sección se avanza sobre el estudio de las diferentes dimensiones del poder, relevando las dificultades que evidencian las concepciones dominantes en la disciplina para dar cuenta de la totalidad de estas y su interrelación. De este modo, una vez reconocidas las limitaciones que enfrentan los abordajes tradicionales, en el cuarto apartado se destaca la importancia de considerar algunas premisas constructivistas para 
optimizar los estudios sobre el poder. Por último, se dedica un espacio final a las conclusiones.

\section{LOS ANÁLISIS DEL PODER EN LA} PERSPECTIVA REALISTA

El concepto de poder adquiere una enorme centralidad en los análisis del realismo y constituye el principal determinante para entender y explicar la dinámica internacional desde esta perspectiva. A pesar de esta generalidad, es preciso aclarar que existen diferentes formas de abordar el concepto al interior del realismo (Schmidt, 2007), que se relacionan con sus distintas vertientes -realismo clásico, neorrealismo, realismo defensivo y ofensivo, y realismo neoclásico-.

Es posible identificar concepciones más sofisticadas como la de Raymond Aron, quien reflexiona sobre las relaciones internacionales contemplando una perspectiva sociológica, ausente en el resto de los autores realistas. Este autor no se queda solo con las definiciones del poder centradas en la posesión de recursos, sino que lo aborda como un fenómeno mucho más complejo, destacando su carácter relacional. Según Aron (1962:99), «el poder de una persona o de una colectividad no puede ser medido rigurosamente, debido a la variedad de objetivos de su comportamiento y de los medios que utiliza». A su entender, "en el campo de las relaciones internacionales, el poder es la capacidad que tiene una unidad política de imponer su voluntad a las demás. En pocas palabras, el poder político no es un valor absoluto sino más bien una relación entre hombres». Esta definición conduce a Aron (1962:100) distinguir entre «los recursos o la fuerza militar de la colectividad, que pueden ser evaluados colectivamente y el poder propiamente dicho, que en cuanto relación humana no depende solo de capacidades materiales e instrumentos.»

Por su parte, Hans Morgenthau -considerado uno de los padres del realismo clásico-, en su obra Política entre las Naciones (1948), también reconoce el carácter relacional del poder, entendiéndolo más bien como un efecto y no solo en términos posesivos. Influenciado por los trabajos de Weber, Morgenthau (1948:51) define el poder como «el control del hombre sobre las mentes y acciones de otros hombres» a lo cual agrega: «el poder político consiste en una relación entre los que lo ejercen y aquellos sobre los cuales es ejercido». Sin embargo, estas definiciones más complejas y sofisticadas en su esencia, no fueron profundizadas y se desdibujaron luego, producto del énfasis que este autor colocó sobre la posesión de recursos al inventariar los elementos del poder nacional -en un esfuerzo por cuantificar y hacer observable en términos positivistas esta importante variable- ${ }^{1}$. Si bien su enfoque atribuye cierta primacía a los factores materiales, y entre estos fundamentalmente a la capacidad militar y económi-

1 Al respecto ver Morgenthau (1948:215294). 
ca, también procura contemplar y dar cabida a otros aspectos importantes.

Cuando procede a inventariar los elementos del poder nacional, Morgenthau (1948:215-294) contempla factores materiales e inmateriales, destacando entre estos últimos la índole nacional, la moral nacional, la calidad de la diplomacia, la calidad del gobierno, la opinión pública. A su vez, el autor distingue a la calidad de la diplomacia como el más importante de todos los factores que entran en la formación del poder de una nación, aunque reconoce que es de naturaleza inestable. El resto de los factores constituye la materia prima con la cual es confeccionado el poder de la nación.

Por su parte, Aron también realiza consideraciones sumamente importantes sobre los elementos de poder que van más allá de lo meramente material. Según este autor el poder no es susceptible de una medición exacta y en tal sentido propone tomar en consideración tres elementos, a saber, el espacio/escenario de acción, los recursos materiales y el conocimiento que permite transformarlos y por último, la capacidad de acción colectiva (Aron, 1962:108-111). Resulta evidente que en el pensamiento de Aron e incluso del propio Morgenthau, la naturaleza de la realidad investigada, es decir la ontología del poder, se presenta sumamente compleja y difusa, por lo cual requiere un abordaje igualmente sofisticado. El intento de combinar elementos cualitativos y cuantitativos para mejorar su comprensión generó dificultades a la hora de la observación y medición del poder.

Una concepción más rudimentaria y simplista se impuso con la fuerza del pensamiento neorrealista -parte medular del mainstream disciplinar-, que surgió de la mano de Kenneth Waltz, con su obra Teoría de la Política Internacional (1977). Waltz se propuso sistematizar los aportes del realismo y fortalecerlo a partir de la elaboración de una teoría de la política internacional rigurosa y con carácter científico -a su entender ausente hasta el momento-. Tal como advierte Sodupe (2003:8283), la definición que brinda Waltz de teoría como explicación de regularidades, encaja plenamente con la de las ciencias naturales. En línea con el positivismo que impregna su producción, Waltz reconoce que los objetos de estudio de las ciencias naturales y las ciencias sociales son diferentes, pero sostiene la unidad del método. La posición epistemológica es clara y marcada en este autor, lo que influye de manera considerable en su concepción del poder.

Las concepciones más sofisticadas presentes en Morgenthau y en Aron fueron marginadas. Waltz -de acuerdo con su forma de entender las funciones de la teoría-, buscó simplificar y abandonó los desarrollos que centraban la atención en los aspectos cualitativos y dificultaban, entre otras cosas, la cuantificación, la medición y consecuentemente su observación. De este modo, Waltz rescató del realismo clásico el énfasis en lo material, en lo concreto. 
Sobre estas bases definió el poder estrictamente en términos de la posesión por parte de agentes individuales -los Estados- de recursos materiales -militares y económicos-.

El análisis de Waltz es eminentemente cuantitativo, se abstrae de todo, salvo de las capacidades de los actores -entendidas en el sentido enunciado anteriormente-, en tal sentido, «lo que emerge es un cuadro posicional, una descripción general de la disposición de una sociedad trazado en términos de la ubicación de las unidades y no en términos de sus cualidades» (Waltz, 1977:147). La noción relacional del poder está completamente ausente en el pensamiento de este autor, ya que se centra en las capacidades materiales de manera más excluyente aún que el realismo clásico. Claramente, la hegemonía positivista en términos epistemológicos, muy fuerte en la disciplina de las relaciones internacionales en ese entonces, condicionó de manera estricta las elecciones ontológicas hacia el materialismo y el individualismo.

Más allá de las diferencias apuntadas, tal como advierte Schmidt (2007:61), existe cierto grado de consenso entre los realistas de las diferentes vertientes en torno a cómo definir el poder. Si bien autores como Morgenthau y Aron procuraron reflejar una visión del poder tanto en términos relacionales como en términos de la posesión de recursos, lo cierto es que la mayoría de los realistas adoptan esta última, concentrándose en los recursos materiales. De este modo, en términos generales, para los realistas el poder es algo que se posee, se puede acumular y es perfectamente medible y cuantificable mediante la utilización de diferentes indicadores, que pueden agregarse en un único indicador de poder.

La definición del poder resultante se muestra concisa, pero al mismo tiempo elemental y reduccionista. Tal como destacan Sterling Folker y Shinko (2007:244), según como lo entienden los realistas, el poder opera en la superficie. En muchas ocasiones, la concepción dominante adoptada por el mainstream disciplinar resulta de escaso valor analítico y limita la comprensión de la política internacional contemporánea.

\section{LOS CUESTIONAMIENTOS A LA CONCEPCIÓN DEL PODER EN TÉRMINOS DE RECURSOS}

Como se mencionó en el apartado anterior, definir el poder como sinónimo de la posesión de recursos permite presentarlo como algo concreto, observable y medible, lo cual facilita los cálculos de quienes formulan la política exterior, en tanto que se establece una relación directa y lineal entre los recursos que un Estado posee y la posibilidad de lograr resultados deseados. Sin embargo, este abordaje representa una simplificación excesiva de la naturaleza del concepto y por tanto de la realidad internacional.

Es posible encontrar situaciones en que Estados con vastos recursos no 
logran alcanzar con éxito sus objetivos. Esta paradoja puede explicarse por diferentes causas, tales como la dificultad para convertir el poder potencial (recursos) en poder real y la pérdida de fungibilidad, que impide pensar el poder con un rol análogo al rol del dinero en la economía. Estas cuestiones son subestimadas cuando los análisis del poder se circunscriben de manera exclusiva a los recursos que se poseen, sin referencia a los factores y variables que intervienen en su movilización y utilización. De este modo, es preciso complementar este enfoque con un abordaje relacional del concepto, tal como lo destacaron Morgenthau y Aron entre otros.

En su obra Poder e Interdependencia (1977), Keohane y Nye reconocen que la naturaleza del poder en las relaciones internacionales se ha vuelto más compleja, producto de los avances tecnológicos y la aparición de nuevos tipos de poder en un contexto de creciente interdependencia y globalización ${ }^{2}$. Según los autores, «dado que la fuerza militar es ineficaz frente a ciertos problemas, la noción convencional de poder carece de precisión. Por lo tanto, pueden llegar a ser necesarios distintos conceptos de poder para enfrentar problemas diversos» (Keohane y Nye, 1977:22).

2 Otro aspecto importante sobre el cual llaman la atención los autores tiene que ver con la difusión del poder, entre otras cosas como consecuencia del surgimiento de actores no estatales en la política internacional, los cuales además operan de acuerdo con una lógica diferente de la estatal, agregando así mayor complejidad.
En la política mundial existen diferentes áreas de cuestiones en las cuales los países no se encuentran posicionados de la misma manera y es posible que aparezcan distintos liderazgos, producto de que un actor sea débil en un área pero fuerte en otra. La fuerza militar puede no ser útil y eficaz para solucionar una cuestión económica o ecológica. Esto se vincula también al carácter multidimensional del concepto, lo cual hace posible que el poder aumente en una dimensión al tiempo que decae en otra (Baldwin, 2002:178). Esta característica es subestimada desde la perspectiva realista.

Por estas razones, desde el enfoque de la interdependencia es de vital importancia contextualizar los usos del poder y pensarlo en los espacios específicos en que se pretende ejercer, y habrá situaciones y cuestiones en que los recursos de poder disponibles serán más efectivos que en otras. En función de estas apreciaciones, Keohane y Nye identifican dos dimensiones centrales para optimizar los análisis del poder en un contexto interdependiente, la sensibilidad y la vulnerabilidad. Estas categorías permiten evaluar los diferentes grados de interdependencia y colocar en una medida más justa su impacto sobre las relaciones de poder.

La sensibilidad implica los grados de respuesta que tiene un actor frente al impacto de situaciones externas que pueden afectarlo, mientras que la vulnerabilidad tiene que ver con la disponibilidad relativa y los costos de las alternativas que los actores deben 
encarar. De este modo, claramente la sensibilidad será menos importante que la vulnerabilidad para proporcionar recursos de poder a los actores, ya que si un Estado puede modificar sus políticas y encontrar alternativas a un bajo costo (baja vulnerabilidad), la sensibilidad de un actor nos dice muy poco sobre su poder (Keohane y Nye, 1977:26-29).

De este modo, resulta evidente la imposibilidad de determinar si un actor tiene poder solo en función de los recursos que posee, es preciso definir "poder para qué». Se debe especificar quienes están involucrados en una relación de poder, así como también qué áreas de cuestiones son consideradas (Nye, 2011:6). Este tipo de análisis permite a un Estado evaluar qué tipos de recursos resultan de mayor utilidad y cuál es la mejor forma de utilizarlos para ejercer poder en un contexto determinado. De acuerdo con todo lo desarrollado, no es posible ignorar el carácter relacional del poder. Un análisis concentrado solo en la posesión de recursos resulta insuficiente, es preciso contemplar los diferentes aspectos que mediatizan su utilización y su traducción efectiva o no en poder real.

\section{LAS DIMENSIONES DEL PODER}

El reconocimiento del poder como un concepto relacional conduce a la necesidad de distinguir las diferentes dimensiones en las cuales opera el poder. En el campo de la ciencia política es posible distinguir tres dimensiones del poder perfectamente aplicables a las relaciones internacionales (Berenskoetter, 2007; Nye, 2011). La primera de ellas, desarrollada por Dahl (1961), hace referencia a la capacidad de comandar cambios mediante un ejercicio directo del poder por un actor sobre otro. La segunda, advertida por Bachrach y Baratz (1963), alude a controlar agendas y así limitar las alternativas de otros actores: aquí el poder opera de manera más indirecta. Por último, la tercera dimensión, introducida por Lukes (2005), se vincula a establecer y moldear preferencias: aquí el poder opera sin necesidad de que exista un conflicto de intereses, aunque existe un aparente consenso es un poder de tipo estructural.

Tal como se evidenció en los dos primeros apartados, los académicos de las relaciones internacionales comúnmente suelen concentrarse en la primera dimensión del poder, sin avanzar lo suficiente en las restantes, con todas las dificultades que esto implica. Las concepciones dominantes en la disciplina centran la atención en el poder que ejerce un actor individual sobre otro, de manera directa y explícita, en función de los recursos que posee. Esta forma de pensar el poder impide alcanzar una comprensión profunda del concepto.

El enfoque de la interdependencia realiza ajustes interesantes a las concepciones realistas del poder, reconociendo su naturaleza cambiante. Si bien su análisis permanece bastante ligado a la primera dimensión, al mismo tiempo abre las puertas para avanzar hacia 
la segunda, fundamentalmente como consecuencia del énfasis colocado en las instituciones y los regímenes internacionales. Desde la perspectiva institucionalista, las capacidades relativas de poder siguen siendo muy importantes -al igual que en el realismo-, sin embargo, al mismo tiempo se subraya la importancia de las instituciones como espacios aptos para canalizar la cooperación y mitigar los efectos de la anarquía.

Si bien la principal preocupación del institucionalismo radica justamente en explorar las posibilidades de cooperación entre los Estados, la cuestión del poder está presente. La cooperación no está exenta de relaciones de poder que la condicionan y le dan forma estas no deben ser ignoradas ni subestimadas. En tal sentido, es importante no caer en la simplificación de pensar que las instituciones anulan por completo las asimetrías de poder y determinan el comportamiento de los Estados tan solo por formar parte de ellas (Creus, 2011:67). No obstante, esto no implica caer en el otro extremo y negar que las instituciones puedan afectar y condicionar el comportamiento de los Estados. De este modo, desde la citada perspectiva, es posible concebir las instituciones como ámbitos que mediatizan los usos del poder y moldean su ejercicio. En consonancia, algunos teóricos como Nye (2004), identifican en las instituciones un recurso de poder.

Por su parte, Baldwin (2002: 187) destaca que «el poder puede ser ejercido en la formación y el mantenimiento de instituciones, a través de estas, en y entre las mismas. Las instituciones pueden reflejar las relaciones de poder, constreñirlas o proveer las bases para su existencia». A diferencia del realismo, el institucionalismo sostiene que las instituciones pueden ser un ámbito útil y eficaz para ejercer poder, para limitar el poder de otros, o incluso para legitimar el propio. El rol de las instituciones siempre fue subestimado por los enfoques realistas -fundamentalmente desde el neorrealismo-, o en el mejor de los casos reducido y subordinado completamente a las capacidades de poder de los Estados participantes. ${ }^{3}$

Así pues, un actor puede movilizar ideas y preferencias en el seno de las instituciones de modo tal que le sean favorables, limitando de esa forma las opciones de otros actores. Tal como señala Berenskoetter (2007: 7-8), el poder aquí y a diferencia de lo que ocurre en la primera dimensión, opera de una manera más sutil e indirecta, a partir de la habilidad de un actor para influenciar y controlar la agenda de una institución en contra de otro actor y a favor de sus intereses.

En línea similar, también es posible incorporar dentro esta dimensión los esfuerzos de los Estados por moldear la estructura de los mercados de un modo tal que los favorezca y mejore su posición relativa en estos, por ejemplo mediante la manipulación del acceso a ellos mediante tarifas, cuotas o licen-

3 Con respecto a esta cuestión, ver Waltz (2000). 
cias, entre otros instrumentos (Nye, 2011:54).

Otra conceptualización interesante que intenta avanzar más allá de la primera dimensión del poder propia de los abordajes tradicionales, es la concepción de poder blando acuñada y sistematizada por Nye (1990; 2004). El término hace referencia a la habilidad de un Estado para influir en el comportamiento de otros mediante la cooptación y la atracción, en lugar de recurrir a la coerción o a la implementación de pagos o compensaciones, que son acciones propias del poder duro. En cuanto al poder entendido como posesión de recursos, si bien la relación es imperfecta, el poder blando emana primariamente de recursos no materiales, tales como la cultura, los valores y las políticas internas y el estilo y la sustancia de la política exterior (Nye, 2004: 6-8).

Nye alude a la capacidad de establecer una agenda determinada y atraer a otros como componente importante del poder blando -estaríamos en la segunda dimensión-, al mismo tiempo que advierte que «el poder blando descansa en la habilidad de formar las preferencias del otro" (Nye, 2004:5). Es en este punto justamente donde Nye da un paso más allá en la conceptualización del poder y se acerca a la denominada tercera dimensión. Tal como se mencionó, la tercera dimensión del poder hace referencia a formar y moldear preferencias.

Para algunos autores como Lukes (2007:95-97), la concepción del poder adoptada por Nye continúa centrándose demasiado en los agentes individuales y el uso por estos de un conjunto determinado de recursos, teniendo dificultades para dar cuenta de las fuerzas estructurales $^{4}$. Nye (2011:16) por su parte reconoce el énfasis que atribuye al agente, pero advierte que igualmente considera fuerzas estructurales aunque en su análisis no incluya todos los aspectos de la estructura. A su entender, "la segunda y la tercera dimensión del poder incorporan aspectos estructurales...pero también dejan espacio para focalizar en los agentes que realizan elecciones, aún constreñidos por fuerzas estructurales» (Nye, 2011:242).

Más allá de estas sutilezas teóricas, Nye (2011:16-18) destaca la importancia de articular una concepción que permita dar cuenta de las diferentes dimensiones del poder, para tener una visión completa de la realidad internacional y las relaciones de poder que se generan. En coincidencia con este autor, se entiende que al incorporar la segunda y la tercera dimensiones - a menudo olvidadas e incluso subestimadas en los análisis de la política internacional-, es posible advertir entre otras cosas la importancia de las narrativas y los discursos, que forman preferencias y consecuentemente moldean el ambiente internacional ${ }^{5}$. No obstante, en este

$4 \quad$ En torno a estos debates es interesante también ver el aporte de Guzzini (1993) y su noción de «poder impersonal».

Para Nye (2011:19-20) «en una era de información, las estrategias de comunicación se vuelven más importantes 
punto es preciso ser cuidadosos y no perder de vista la relación entre estas dimensiones del poder, entendido en sentido relacional y la posesión de ciertos recursos que sirven como sustento. En tal sentido, es conveniente recordar que los diferentes actores en el plano internacional no se encuentran en las mismas condiciones, ni disponen de las mismas capacidades para establecer narrativas socialmente aceptadas.

Más allá de todos los aportes citados, resulta evidente que las teorías tradicionales de las relaciones internacionales encuentran dificultades para pensar y reflexionar sobre el poder en sus diferentes dimensiones y precisan el auxilio de otras perspectivas que complementen sus enfoques. Analizar la tercera dimensión del poder exige algo más que explicar las relaciones de poder, en tanto que exige entrar en el campo de la comprensión. Es en este terreno donde los abordajes tradicionales flaquean y requieren incorporar premisas de corte interpretativista para reforzar y optimizar sus análisis. En este sentido, resulta importante destacar el aporte del constructivismo -en sus diferentes formulaciones ${ }^{6}$, , que ha logrado

y los resultados no son moldeados meramente por aquellos cuyo ejército triunfa, sino más bien por aquellos cuya historia triunfa». Para el autor, esto se presenta muy claro en el caso de la guerra de Irak, donde las agencias de información y comunicación tales como CNN, BBC y Al Jazeera, jugaron y aún juegan un rol central en este aspecto.

6 El constructivismo no constituye una formulación homogénea, en su interior es considerable influencia en la disciplina, siendo útil y necesario reparar en algunos de sus principales aspectos.

El aporte del constructivismo

Desde el constructivismo se entiende la realidad como socialmente construida. Su irrupción en el campo de las relaciones internacionales plantea la necesidad y la importancia de proceder a un abordaje social de la política internacional, donde el comportamiento de los Estados no se explica por las capacidades de estos sino más bien por las creencias, las ideas y las identidades. En tal sentido, resulta evidente la recuperación de una perspectiva sociológica para el estudio de las relaciones internacionales. En lo que respecta al concepto de poder, Wendt (1999: 1) señala que «el poder está constituido primariamente por ideas y contextos culturales, más que por fuerzas materiales brutas», pero no avanza más allá de esta afirmación.

En términos generales, se puede decir que el constructivismo abraza una ontología idealista y holista. Idealista a partir de que rescata y resalta el impacto de las ideas sobre las fuerzas materiales, y holista dado que asume que las estructuras tienen efectos constitutivos sobre las propiedades de los agentes (Wendt, 1999:22-33; Sodupe, 2003:63-67), aunque estos últimos también afectan a la estructura. Cabe destacar que tal como advierte Sodupe (2003:176):

posible identificar diferentes formulaciones, al respecto ver Zehfuss (2004), Guzzini y Leander (2006). 
Para el constructivismo, del mismo modo que las estructuras sociales son ontológicamente dependientes de y, por tanto, constituidas por las prácticas y formas de entender de los agentes, los poderes causales e intereses de estos agentes, a su vez, están generados y, por tanto, explicados por las estructuras. En definitiva, agentes y estructuras, aunque ontológicamente distintos, son entidades mutuamente constituidas. Cada una en cierto sentido afecta a la otra: están co-determinadas.

De este modo, las mencionadas elecciones ontológicas permiten al constructivismo proceder a un análisis más sofisticado del poder, capaz de abordarlo en su tercera dimensión. Es posible advertir que desde la perspectiva constructivista las capacidades de poder -materiales y no materiales-adquieren pleno significado solo en el marco de la comunicación intersubjetiva. El propio Wendt reconoce que si bien la distribución de poder puede afectar los cálculos de los Estados, la forma en la que lo haga depende de los entendimientos y expectativas intersubjetivas, de la distribución de conocimiento que constituye sus concepciones de sí mismo y del otro (1992:397). Es preciso determinar cuál es la estructura social predominante en las diferentes relaciones de poder. ${ }^{7}$ Esto

7 Wendt (1999: 246) en términos generales identifica tres tipos ideales de estructuras sociales para explicar y comprender la dinámica de la política internacional: la hobbesiana, la lockeana y la kantiana, las que representan tres tipos diferentes de cultura: la enemistad, la rivalidad y la amistad. no significa que para el constructivismo los recursos no cuentan, sino sencillamente que estos por si solos no tienen la capacidad explicativa que pretenden los abordajes tradicionales. No obstante, cabe destacar que mientras en lo que respecta al eje estructura-agente Wendt opta por una vía intermedia en el que ninguno predomina sobre el otro, en lo que respecta al eje materialismoidealismo, las ideas predominan.

El constructivismo no puede concebir el poder solo en términos de recursos, en tanto que desde su óptica, los agentes actúan hacia los objetos en función de los significados que les asignan. El poder es entonces el producto resultante de los significados compartidos en las relaciones sociales. En tal sentido, establecer significados o narrativas socialmente aceptadas constituye un claro ejercicio de poder, en tanto que condiciona la forma en la cual los agentes piensan y actúan, dicho de otra manera, moldea sus preferencias. En función de estas afirmaciones, Guzzini (2007) llama la atención sobre la importancia de proceder a un análisis constructivista del poder. Para este autor, es necesario cambiar el análisis conceptual y en lugar de insistir en preguntarse qué significa el poder, preguntarse qué implica el uso del concepto de poder, de este modo es posible observar cabalmente su importancia (Guzzini, 2000; 2007). Este giro permite, a partir del concepto de poder, establecer un vínculo entre la construcción de conocimiento y el orden social (Guzzini, 2000:172). 
Para el autor, este vínculo puede verse con claridad cuando se ponen ciertas etiquetas, por ejemplo «cuando el FMI le coloca a un país la categoría de insolvente, dicho país es despojado de poder en sus relaciones sociales. Otros actores financieros cambiarán su comportamiento en consonancia» (Guzzini, 2000:172). A este ejemplo pueden adicionarse muchos otros, uno muy similar, siguiendo en el plano de las finanzas internacionales, resulta sin dudas del rol que juegan las calificadoras de riesgo. En estos casos, nuevamente, al igual que cuando se hizo referencia a la construcción de narrativas socialmente aceptadas, es preciso recordar que no todos los actores tienen la misma efectividad a la hora de colocar etiquetas o establecer significados. De este modo, no debe perderse de vista la dimensión material que muchas veces respalda dichas construcciones. Más allá de esto, prestar atención a estas particularidades del poder permite a los diferentes actores percibir su dinámica real de funcionamiento y responder en consecuencia, contemplando alternativas que a priori parecían excluidas o inviables. ${ }^{8}$

$8 \quad$ En la década de los años noventa por ejemplo, muchos Estados abrazaron la narrativa del «Consenso de Washington» $\mathrm{y}$ adoptaron estrictamente las recetas neoliberales y las recomendaciones del FMI: ninguna alternativa parecía viable por fuera de dichos parámetros. No obstante, hubo algunos Estados que aun sin los recursos suficientes como para sostener una narrativa alternativa, encontraron grietas y márgenes de
Guzzini (2007:35) entiende que «un análisis conceptual del poder en términos de su significado es parte de la construcción social del conocimiento, más aún, la definición/asignación de poder es en sí misma un ejercicio de poder» (2007:35). El poder no es otra cosa más que una construcción social. Como puede verse en este breve desarrollo, a diferencia de lo que ocurre desde otros enfoques, la tercera dimensión del poder está claramente incorporada en la perspectiva constructivista y adquiere una relevancia crucial para entender la política internacional. Tomar en

maniobra que le permitieron matizar algunos aspectos de la narrativa dominante. Asimismo, otro ejemplo puede encontrarse en los procesos de reestructuración de deuda soberana, donde la narrativa dominante prescribe la implementación de fuertes políticas de ajuste para resolver los problemas. Sin embargo, existen casos como el de Argentina, que luego de su crisis de 2001 y la declaración de default inició un arduo proceso de renegociación de su deuda externa en el cual, aprovechando el descrédito de las instituciones financieras internacionales y un contexto internacional benévolo, logró articular una narrativa alternativa que priorizaba el crecimiento como variable para salir de la crisis. Si bien esta narrativa no logró erigirse en dominante a nivel global -las respuestas a la crisis europea son el claro ejemplo-, sirvió para el caso particular y para demostrar que otras políticas eran posibles, sin necesariamente "caerse del mundo" como muchos presagiaban. Cabe destacar, que el buen desempeño de la economía, los elevados índices de crecimiento y la suba de los precios de los commodities, resultaron fundamentales para dar sustento material a la citada narrativa. 
consideración tales premisas, sin dudas resulta enriquecedor, esto independientemente de la perspectiva teórica que se adopte como predominante.

\section{Conclusión}

El recorrido teórico propuesto, sin pretender ser exhaustivo ni mucho menos, procuró dar cuenta de algunos de los principales debates en torno al concepto de poder en las relaciones internacionales, con el simple objetivo de poner de manifiesto su complejidad y la necesidad de proceder a abordajes más sofisticados. Tal como lo advierten muchos autores, es menester romper con la tentación simplificadora de definir el poder exclusivamente en términos de la posesión de recursos. Un avance fundamental radica en reconocer su carácter relacional y explorar las diferentes dimensiones en las que opera.

Las formas tradicionales de entender el poder en las relaciones internacionales resultan insuficientes para desentrañar la complejidad de la política mundial, dado que aún cuando logran avanzar hacia el reconocimiento de su carácter relacional, permanecen demasiado concentradas en la primera dimensión del poder. Tal como se mencionó, esto se debe en gran parte al predominio del positivismo en términos epistemológicos y las consecuentes elecciones ontológicas hacia el materialismo y el individualismo, todo lo cual conduce a un análisis más superficial de las relaciones de poder y su dinámica, concentrándose en lo inmediatamente tangible y observable, sin considerar otras variables y factores que complejizan la cuestión. Claro está que esta es solo una parte de la historia, o mejor dicho tan solo una dimensión del poder.

Si bien desde algunos enfoques tales como la interdependencia o el institucionalismo se brindan concepciones más sofisticadas, lo cierto es que muestran dificultades para avanzar hacia una comprensión más profunda del poder que incluya y pueda dar cuenta cabalmente de la tercera dimensión: para esto es preciso ampliar el horizonte teórico. Es aquí donde el constructivismo proporciona aportes interesantes en función de su abordaje de la política internacional como una realidad socialmente construida. Además, las premisas constructivistas se presentan útiles para matizar las concepciones centradas de manera excluyente en el agente y dar cabida a los factores estructurales.

Tal como se evidenció a lo largo del trabajo, el poder en la política internacional presenta diversas alternativas para su ejercicio y construcción, todo depende de la óptica teórica desde la cual se reflexione al respecto. Según las posiciones ontológicas y epistemológicas que priman en un enfoque teórico determinado, se destacan distintos aspectos y dimensiones del poder. No se trata entonces de eliminar los abordajes tradicionales, sino de incorporar nuevos enfoques según los aspectos y dimensiones del poder que se pretenda analizar. Asimismo, no se trata de hacer primar una dimensión del poder 
por encima de las restantes. El desafío radica en entenderlas de manera integrada, no para lograr una concepción del poder unificada, sino más bien con el propósito de entender cuál es la interrelación existente entre las distintas dimensiones y cómo opera el poder en cada una de ellas. Solo contemplando estas cuestiones es posible hacer inteligible un concepto sumamente complejo y problemático. Los análisis del poder ya no pueden quedar confinados a los supuestos de la teoría realista y la ampliación del horizonte teórico resulta tan necesaria como inevitable.

\section{BibliogRAFÍA}

Aron, Raymond (1962), Paz e Guerra entre as naçoes, trad. Sérgio Bath (1 a. edição) Brasília, Editora Universidade de Brasília, Instituto de Pesquisa de Relações Internacionais, São Paulo, Imprensa Oficial do Estado de São Paulo, 2002.

Bachrach, Peter y Baratz, Morton (1963), "Decisions and Nondecisions: An Analytical Framework", American Political Science Rewiew, vol 57, n³, pp. 632-642.

Baldwin, David (2002), «Power and International Relations», en Carlsnaes W, T. Risse-Kappen y B. Simmons B, Handbook of international relations, Londres, SAGE publications Ltd, pp. 177-191.

Berenskoetter, Félix (2007), «Thinking about power», en Berenskoetter, Félix y, M. J. Williams, Power in world politics, Nueva York, Routledge, pp. 1-22.

Creus, Nicolás (2011), «La autonomía en la política exterior argentina frente a un desafío inexorable: reflexionar sobre el poder», en Miranda, Roberto (comp), Política exterior. Conceptos y enfoques en torno a Argentina, Rosario, Ediciones Pia, pp. 49-76.
Dahl, Robert (1957), "The Concept of Power", Behavioral Science, vol 2, n³, pp. 201-215.

Guzzini, Stefano (1993), «Structural power: The limits of neorealist power analysis», International Organization, ${ }^{\circ} 47,3$, pp. 443-478.

Guzzini, Stefano (2000), «A reconstruction of constructivism in International Relations", European Journal of International Relations, $\mathrm{n}^{\circ}$ 6, 2, pp. 147-182.

Guzzini, Stefano (2007), «The concept of power: a constructivist analysis", en Berenskoetter, Félix y Williams, M. J., Power in world politics, NuevaYork, Routledge, pp. 23-42.

Guzzini, Stefano y Anna Leander, (2006), Constructivism and International Relations. Alexander Wendt and his critics, Nueva York, Routledge.

Keohane, Robert y Nye, Joseph (1977), Poder e interdependencia, Buenos Aires, Grupo Editor Latinoamericano (GEL), 1988.

Lukes, Steven (2005), Power: A Radical View, 2a. ed., Nueva York, Palgrave Macmillan.

Lukes, Steven (2007), «Power and the battle for hearts and minds: on the bluntness of soft power», en Berenskoetter, Félix y M. J. Williams, Power in world politics, Nueva York, Routledge, pp. 83-97.

Morgenthau, Hans (1977), A política entre as naçoes. A luta pelo poder e pela paz, trad. Oswaldo Biato Brasília, Editora Universidade de Brasília, Instituto de Pesquisa de Relações Internacionais, São Paulo, Imprensa Oficial do Estado de São Paulo, 2003.

Nye, Joseph (1990), Bound to Lead, Nueva York, Basic Books.

Nye, Joseph (2004), Soft Power: The means to success in world politics, Nueva York, Public Affairs.

Nye, Joseph (2011), The future of power, Nueva York, Public Affairs.

Schmidt, Brian (2007), «Realist conceptions of power», en Berenskoetter, Félix y M.J. Williams, Power in world politics, Nueva York, Routledge, pp. 43-63. 
Sodupe, Kepa (2003), La teoría de las Relaciones Internacionales a comienzos del siglo XXI, ed. Universidad del País Vasco.

Sterling-Folker, Jennifer y Rosemary (2007), "Discourses of power: traversing the realist-postmodern divide», en Berenskoetter, Félix y M. J. Williams, Power in world politics, Nueva York, Routledge, pp. 244-264.

Waltz, Kenneth (1977), Teoría de la Política Internacional, Buenos Aires, GEL, 1988.

Waltz, Kenneth (2000), "Structural Realism after de Cold War", International Security vol 25, n²1, pp 5-41.

Wendt, Alexander (1992), «Anarchy is what states make of it: the social construction of power politics», International Organization vol 46, n², pp. 391-425.

Wendt, Alexander (1999), Social Theory of International Politics, Reino Unido, Cambridge University Press.

Zehfuss, Maja (2004), Constructivism in International Relations. The politics of reality, Nueva York, Cambridge University Press. 